\title{
Programas de Pós-graduação stricto sensu em enfermagem no Brasil e seus direcionamentos teórico-filosóficos como componente curricular
}

\author{
Stricto sensu postgraduate programs in nursing in Brazil and its theoretical-philosophical \\ directions as a curricular component \\ Postgrados estricto sensu em enfermeira em Brasil y sus orientaciones teórico-filosóficas como
} componente curricular

\author{
Ianka Cristina Celuppi \\ ORCID: https://orcid.org/0000-0002-2518-6644 \\ Universidade Federal de Santa Catarina, Brasil \\ E-mail: iankacristinaceluppi@gmail.com \\ Paulo Roberto Boeira Fuculo Júnior \\ ORCID: https://orcid.org/0000-0002-0288-3965 \\ Universidade Federal de Santa Catarina, Brasil \\ E-mail: paulo.fuculo@hotmail.com \\ Stefhanie Conceição de Jesus \\ ORCID: https://orcid.org/0000-0002-9716-5471 \\ Universidade Federal de Santa Catarina, Brasil \\ E-mail: stefhaniec.j@gmail.com \\ Fernando Henrique Antunes Menegon \\ ORCID: https://orcid.org/0000-0002-8840-4653 \\ Universidade Federal de Santa Catarina, Brasil \\ E-mail: fernandomenegon01@gmail.com \\ Davydson Gouveia Santos \\ ORCID: https://orcid.org/0000-0002-5645-7785 \\ Universidade Federal de Santa Catarina, Brasil \\ Centro Universitário Maurício de Nassau, Brasil \\ E-mail: davydsongouveia@gmail.com \\ Ângela Maria Alvarez \\ ORCID: https://orcid.org/0000-0002-2622-3494 \\ Universidade Federal de Santa Catarina, Brasil \\ E-mail: angela.alvarez@ufsc.br \\ Rosane Gonçalves Nitschke \\ ORCID: https://orcid.org/0000-0002-1963-907X \\ Universidade Federal de Santa Catarina, Brasil \\ E-mail: rosane.nitschke@ufsc.br
}

\begin{abstract}
Resumo
Objetivo: analisar o conteúdo filosófico de disciplinas dos cursos de pós-graduação stricto sensu em enfermagem no Brasil. Método: estudo exploratório, descritivo, do tipo análise documental, realizado de junho a julho de 2019 . Os dados foram coletados em planos de ensino das disciplinas dos cursos de pós-graduação em enfermagem na modalidade acadêmica de instituições de ensino superior no Brasil. Utilizou-se estatística descritiva para a análise dos dados. Resultados: Obteve-se os planos de ensino de 12 programas de pós-graduação em enfermagem. Nas ementas selecionadas, evidencia-se a preocupação em estudar aspectos históricos e as bases da filosofia, através de correntes de pensamentos associadas ao processo de cuidar, a ética e ao raciocínio em enfermagem. Considerações finais: Os programas que abordam a filosofia em suas disciplinas apresentam uma preocupação em discutir com os discentes os aspectos conceituais, históricos e as correntes de pensamento, associadas ao processo de cuidar, à ética e ao raciocínio na enfermagem. A falta de acesso aos planos de ensino evidenciou uma lacuna no ensino na pós-graduação em enfermagem no Brasil.
\end{abstract}

Palavras-chave: Educação de pós-graduação em enfermagem; Filosofia em enfermagem; Pesquisa em enfermagem.

\begin{abstract}
Objective: to analyze the philosophical content of disciplines in postgraduate stricto sensu courses in nursing in Brazil. Method: exploratory, descriptive, documentary analysis, conducted from June to July 2019. Data were collected in teaching plans for the disciplines of graduate nursing courses in the academic modality of higher education institutions in Brazil. Descriptive statistics was used for data analysis. Results: The teaching plans of 12
\end{abstract}


nursing graduate programs were obtained. In the selected menus, the concern with studying historical aspects and the bases of philosophy is evidenced, through currents of thoughts associated with the care process, ethics and reasoning in nursing. Final considerations: Programs that address philosophy in their disciplines are concerned with discussing with students the conceptual, historical aspects and currents of thought, associated with the care process, ethics and reasoning in nursing. The lack of access to teaching plans evidenced a gap in teaching in graduate nursing courses in Brazil.

Keywords: Nursing graduate education; Nursing philosophy; Nursing research.

\section{Resumen}

Objetivo: analizar el contenido filosófico de las disciplinas en cursos de posgrado stricto sensu en enfermería en Brasil. Método: análisis exploratorio, descriptivo, documental, realizado de junio a julio de 2019. Los datos fueron recolectados en planes de enseñanza para las disciplinas de cursos de posgrado en enfermería en la modalidad académica de instituciones de educación superior en Brasil. Se utilizó estadística descriptiva para el análisis de datos. Resultados: Se obtuvieron los planes de docencia de 12 programas de posgrado en enfermería. En los menús seleccionados se evidencia la preocupación por estudiar aspectos históricos y las bases de la filosofía, a través de corrientes de pensamiento asociadas al proceso de cuidado, la ética y el razonamiento en enfermería. Consideraciones finales: Los programas que abordan la filosofía en sus disciplinas se preocupan por discutir con los estudiantes los aspectos conceptuales, históricos y corrientes de pensamiento, asociados al proceso de cuidado, la ética y el razonamiento en enfermería. La falta de acceso a los planes de enseñanza evidenció una brecha en la docencia en los cursos de posgrado en enfermería en Brasil.

Palabras clave: Educación de posgrado en enfermería; Filosofía de enfermería; Investigación en enfermería.

\section{Introdução}

A filosofia tem como essência o questionamento, a reflexão e a ponderação sobre os fenômenos da vida. Assim, podese denominar atitude filosófica aquela relacionada a não aceitar os fatos como óbvios e evidentes, mas buscar investigá-los e questioná-los. Esse questionamento dos fatos e a busca por compreendê-los é determinante para a evolução do conhecimento ao longo da história (Mcewen; Wills, 2015).

A dimensão filosófica do cuidado é permeada por uma questão antropológica: o significado do ser humano. O cuidado consiste na forma como o ser humano está no mundo e representa a relação entre o eu e o outro. Desse modo, por sofrer influências do tempo e do espaço, o cuidado pode ser compreendido como acontecimento histórico e filosófico cuja construção é influenciada pelas características socioculturais dos diferentes povos (Salviano et al., 2016; Sousa; Koerich, 2008).

As abordagens filosóficas em enfermagem estão presentes como componente formativo no conteúdo programático das disciplinas de pós-graduação stricto sensu em enfermagem em nível de mestrado e doutorado, contribuindo para a constituição do campo científico da profissão em relação à formação. Nesse sentido, avança-se na definição de um perfil acadêmico e profissional que inclua o desenvolvimento de competências e habilidades que possam subsidiar a formação reflexiva com ênfase na realidade social e a geração de mudanças nos projetos de pesquisas e consequentemente nos serviços de saúde (Guimarães et al., 2017).

Assim, a evolução do conhecimento científico está atrelada ao desenvolvimento da profissão, a qual converge para o desenvolvimento aprimorado e inovador de sua prática, elemento essencial para a formação no âmbito assistencial, gerencial e educacional, envolvendo ensino, pesquisa e extensão. Assim, acredita-se que as disciplinas que abordam a temática da Filosofia em Enfermagem estimulem o aprimoramento da efetiva construção de discursos reflexivos e atuantes na prática profissional (Schmitz et al., 2016).

Disciplinas relacionadas à antropologia e à filosofia auxiliam no pensamento crítico e humanístico que permeiam discussões sobre as condições humanas, compreendendo os distintos grupos sociais e as relações do cuidado, melhorando as práticas de enfermagem, uma vez que a arte, a ética e a ciência asseguram um cuidado de melhor qualidade (Liberatori, 2019).

O presente estudo emergiu das reflexões suscitadas numa disciplina que aborda as concepções teórico-filosóficas no processo de cuidar em saúde e enfermagem, oferecida em programa de pós-graduação stricto sensu de universidade pública de 
um o estado no sul do Brasil. Apesar dos avanços acerca da temática da Filosofia em Enfermagem e sua influência na formação nos cursos de pós-graduação, a produção científica brasileira sobre esse tema ainda é incipiente.

Diante do exposto, definiu-se como questão de pesquisa: Quais os componentes filosóficos são abordados nas disciplinas dos cursos de pós-graduação stricto sensu em enfermagem no Brasil? Assim, com o propósito de responder à questão acima, definiu-se como objetivo do estudo: analisar o conteúdo filosófico de disciplinas dos cursos de pós-graduação stricto sensu em enfermagem no Brasil.

Os momentos destinados ao estudo filosófico da profissão contribuem para fortalecer uma formação crítico-reflexiva na enfermagem, o que justifica este estudo, enfatizando também sua relevância ao possibilitar conhecer o panorama da abordagem filosófica nos planos de ensino das disciplinas sobre concepções filosóficas dos cursos de pós-graduação em enfermagem, subsidiando possibilidades de estratégias e abordagens. Além disso, o estudo contribui para construir novos conhecimentos e ressignificação da práxis de mestrandos e doutorandos.

\section{Metodologia}

Estudo exploratório, descritivo, do tipo análise documental, realizado de junho a julho de 2019, tendo como objeto as ementas das disciplinas dos cursos de pós-graduação em enfermagem stricto sensu do Brasil.

O estudo foi desenvolvido em duas etapas: a primeira com o intuito de buscar todas as instituições de ensino superior (IES) públicas com programas de pós-graduação em Enfermagem (PPGENF) stricto sensu. Para encontrá-las, foi consultado o Sistema Nacional de Pós-Graduação (SNPG) no portal da Coordenação de Aperfeiçoamento de Pessoal de Nível Superior (CAPES). Uma vez localizadas e listadas, as IES/PPG foram selecionadas, respeitando os critérios de inclusão do estudo, como se vê na Figura 1. 
Figura 1. Busca, seleção e análise das IES.

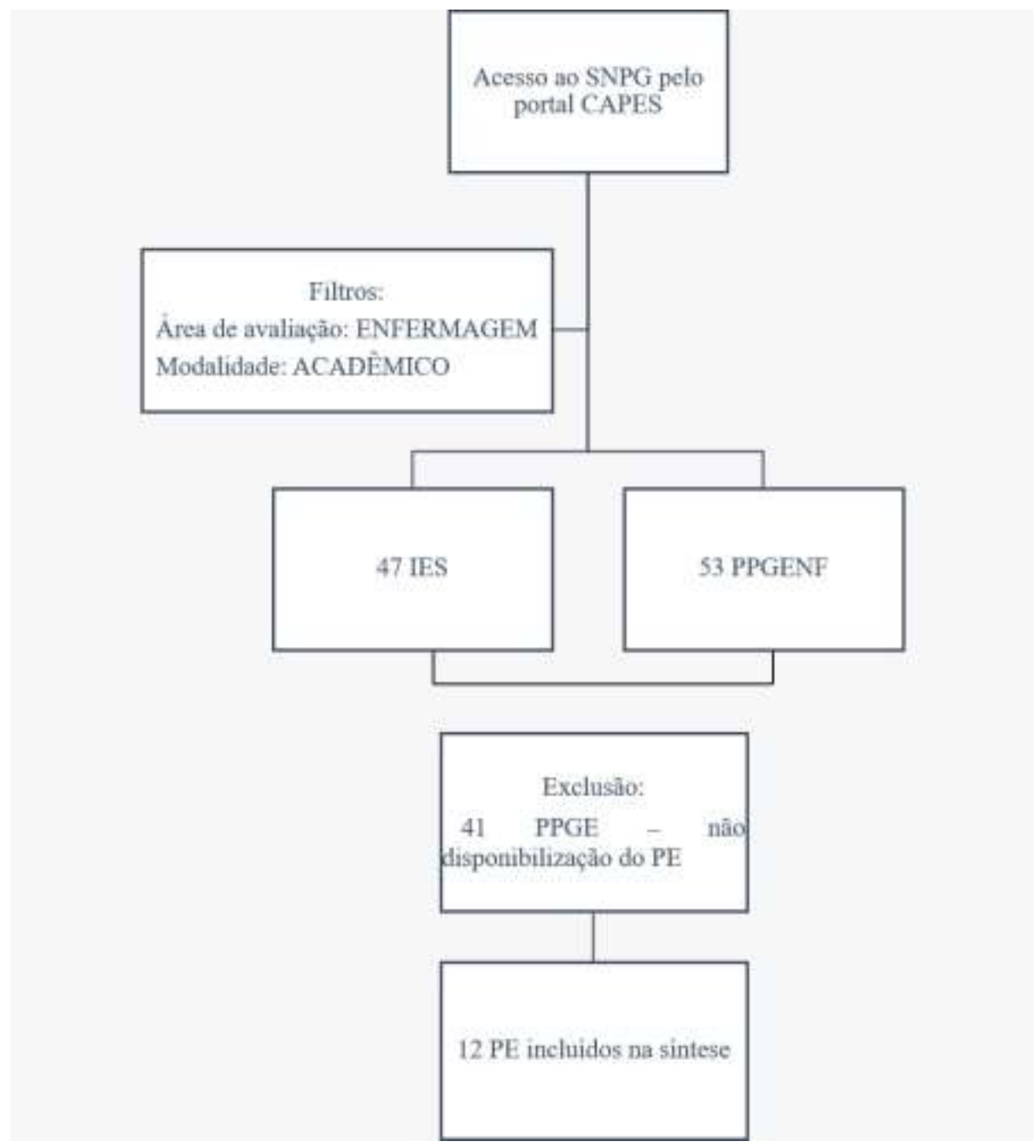

Fonte: Autores. Florianópolis/SC (2019).

Foram incluídas as disciplinas dos cursos de pós-graduação stricto sensu, na área da Enfermagem, modalidade acadêmica de IES em funcionamento no SNPG, identificadas no portal da Coordenação de Aperfeiçoamento de Pessoal de Nível Superior (CAPES) com disponibilidade eletrônica dos Planos de Ensino (PE) na íntegra. Durante a análise dos PE, foram selecionados aqueles que apresentaram estudos filosóficos na enfermagem, identificados pela utilização de palavras-chave presentes na descrição do plano, como "filosofia", "estudo filosófico", "concepções filosóficas", "filosofia do cuidado", dentre outras, com suas semelhanças. Excluíram-se as IES com programas apenas de doutorado e programas sem PE disponível em endereço eletrônico.

Construído pelos autores, o instrumento de coleta de dados contemplou os seguintes aspectos: região do Brasil, estado, sigla da IES, nome completo da IES, nome do programa, área de avaliação, modalidade, nível, nota do programa na CAPES, endereço eletrônico, plano de ensino, matriz curricular, ementa, abordagem de questões filosóficas, carga horária, método de ensino-aprendizagem e conteúdo programático.

As disciplinas foram identificadas, numa busca eletrônica dos PEs de cada PPG, realizada por dois pesquisadores concomitantemente e de forma independente. Calculou-se a intensidade de concordância dessas avaliações pelo coeficiente Kappa (Cohen, 1960). Foi considerada a seguinte classificação: menor que 0,00, pobre concordância; 0,00 - 0,20, leve concordância; 0,21 - 0,40, razoável concordância; $0,41-0,60$, moderada concordância; 0,61-0,80, substancial concordância, e 0,81 - 1,00, quase perfeita concordância (Landis; Kock, 1977). Foi obtido 0,73 para a avaliação dos PE, e 0,80 para a 
avaliação da presença de disciplina, isto é, ambas as avaliações com substancial concordância entre os pares. Discordâncias, portanto, foram eliminadas por um terceiro pesquisador.

Os dados extraídos foram levados ao programa Microsoft Excel®, versão 2019, depois para o SPSS do Windows, versão 21, para estatística descritiva, com frequências absolutas e relativas.

Para fins deste estudo e assegurando o anonimato das instituições, cada IES recebeu um código numérico: a primeira IES foi identificada como IES1, a segunda como IES2 e assim sucessivamente. Por utilizar dados de domínio público, o estudo não foi submetido à apreciação de Comitê de Ética.

\section{Resultados e Discussão}

Foram identificados 53 Programas de Pós-graduação em Enfermagem (PPGENF) de 47 instituições de ensino superior; dois PPGENF foram excluídos por ofertar apenas cursos em nível de doutorado, e 39 por não disponibilizar o PE em endereço eletrônico institucional. Portanto, foram analisados os PE de 12 PPGENF disponíveis.

Cabe destacar o alto índice do coeficiente de Kappa, indicando elevada concordância entre avaliadores. Considerou-se que os avaliadores estavam nivelados num patamar semelhante pelo fato de já terem compartilhado o tema por meio do convívio e das reflexões suscitadas numa disciplina que aborda as concepções filosóficas no processo de cuidar em saúde e enfermagem, contribuindo para que a avaliação seguisse o mesmo padrão.

Do total, seis (50,0\%) PE apresentaram disciplinas com abordagem baseada em concepções filosóficas no processo de cuidar da enfermagem, sendo a maioria desses (três) PPG de IES localizadas na Região Sudeste, seguido de duas na Região Sul e uma localizada no Nordeste do Brasil.

A baixa disponibilidade de PE de disciplinas que abordam as reflexões filosóficas no processo de cuidar revelou uma lacuna do ensino dessa temática na pós-graduação em enfermagem, porquanto uma disciplina de filosofia no contexto acadêmico propicia experiências humanizadoras no cuidado de enfermagem, e a abordagem filosófica visa a uma educação comprometida (Barbosa; Reginato; Gallian, 2015).

Convém salientar ainda a percepção de próprios pós-graduandos ante a necessidade de aprofundamento teórico a fim de propiciar melhor formação ao profissional enfermeiro, com estruturas curriculares que permitem tal proveito (Merino et al., 2018).

Embora o quantitativo de disciplinas tenha sido considerado pequeno em relação ao número de PPG, a carga horária das disciplinas identificadas foi relevante: média de 57 horas.

Quanto aos métodos de ensino-aprendizagem nessas disciplinas, foram identificados diferentes tipos utilizados: leituras e discussões em aulas (apresentado em seis PE), seguida de seminários (apresentado em três PE) e elaboração de material (apresentado em três PE). No entanto, em apenas um de todos o PE avaliados, foram identificados outros métodos de ensino-aprendizagem, como oficinas, aulas expositivas dialogadas e avaliação teórico-prática.

As disciplinas oferecidas nos cursos de pós-graduação têm promovido momentos para conduzir as reflexões filosóficas dos estudantes, predominando o método de leitura e discussão em sala de aula, o que permite uma construção coletiva do conhecimento com base em diferentes perspectivas e pautada nas evidências científicas. Nota-se um distanciamento da educação dogmática, em que o estudante é exclusivamente receptor do conhecimento provindo do professor, não cabendo contestação nem confrontos por meio da troca de argumentos. A educação dogmática não oportuniza, portanto, a construção do conhecimento fundada nas experiências prévias e nos diálogos com os demais alunos (Delors et al., 1998).

Considerando que o saber em saúde se apresenta como instrumento de autonomia profissional, e que as diferentes formas de conhecimento têm contribuído para integrar teoria, pesquisa e prática (Mcewen; Wills, 2015; Salcedo-Álvarez, 
2017), evidencia-se a importância da promoção de reflexões no cenário educacional, onde o aluno mantenha uma relação constante do ensino com o campo de assistência à saúde, agregando valor ao fazer profissional (Merino et al., 2018). O cuidado em saúde, que é o objeto de trabalho da enfermagem, tem profunda sustentação filosófica e sua utilidade é essencialmente prática (Salcedo-Álvarez, 2017).

No seu processo formativo, o enfermeiro precisa ser movido a desenvolver a atividade crítico-reflexiva, haja vista a complexidade e a subjetividade que envolvem o cuidar. Assim, justifica-se integrar concepções filosóficas na formação da pósgraduação, pela necessidade de agregar limitadas abordagens na graduação, cabendo retomar conceitos e aprofundá-los em discussões reflexivas na oportunidade da pós-graduação (Merino et al., 2018). Os conteúdos abordados nas disciplinas de concepções filosóficas estão apresentados no Quadro 1.

Quadro 1. Conteúdo programático abordado nas disciplinas identificadas nos seis PE dos PPG stricto sensu em enfermagem, modalidade acadêmico das IES no Brasil, 2020.

\begin{tabular}{|c|c|}
\hline IES & Conteúdo Programático \\
\hline IES1 & $\begin{array}{l}\text { O pensamento científico. A epistemologia e a história da Ciência. A racionalidade } \\
\text { científica. Correntes teórico-filosóficas. Positivismo, fenomenologia e materialismo } \\
\text { histórico e dialético. O pensamento pós-moderno. A construção do conhecimento na } \\
\text { enfermagem: marcos históricos e conceituais. }\end{array}$ \\
\hline IES2 & Origem e bases teóricas do conhecimento. As principais correntes do pensamento. \\
\hline IES3 & $\begin{array}{l}\text { Concepções filosóficas (ontológicas e epistemológicas) e sociais no processo de cuidar em } \\
\text { saúde e enfermagem: aspectos históricos e filosóficos. Correntes de pensamento na área da } \\
\text { saúde e enfermagem. O saber em saúde e em enfermagem: teoria e prática; perspectivas de } \\
\text { análise do conhecimento; estrutura do conhecimento contemporâneo de enfermagem. } \\
\text { Concepções filosóficas e políticas em saúde e enfermagem. Processo de cuidado em saúde e } \\
\text { enfermagem. A consciência moral e a ética na construção do conhecimento. }\end{array}$ \\
\hline IES4 & $\begin{array}{l}\text { Ética em pesquisa com seres humanos: contexto histórico e situação atual no Brasil e no } \\
\text { mundo. Resoluções nacionais da ética em pesquisa. Ética em pesquisa com seres humanos: } \\
\text { vulnerabilidade, análise crítica dos riscos, benefícios, termo de consentimento livre e } \\
\text { esclarecido. Diretrizes éticas para as atividades científicas. Boas práticas científicas. } \\
\text { Honestidade científica. Fraude acadêmica. Integridade da pesquisa. }\end{array}$ \\
\hline IES5 & $\begin{array}{l}\text { Antecedentes históricos da bioética no Brasil e no mundo. Conceito de Bioética e seus } \\
\text { fundamentos teórico-filosóficos. Análise histórica e filosófica dos principais dilemas } \\
\text { advindos do desenvolvimento técnico-científico; Debate sobre a importância da aplicação } \\
\text { das ciências da vida na sociedade; Discussão de questões éticas em saúde e enfermagem. }\end{array}$ \\
\hline IES6 & $\begin{array}{l}\text { As três operações da inteligência: intuição, juízo e raciocínio. Os instrumentos da razão: } \\
\text { definição, enunciação e argumentação. A estrutura dos argumentos. Avaliando argumentos } \\
\text { científicos. Falácias. Pensamento crítico na enfermagem. }\end{array}$ \\
\hline
\end{tabular}

Fonte: Autores. Florianópolis/SC (2019).

O Quadro 1 apresenta os conteúdos que são abordados nas disciplinas que envolvem concepções filosóficas nas IES, contemplando temáticas como: pensamento científico, correntes filosóficas, construção do conhecimento na enfermagem, ética em pesquisa, práticas científicas, razão, argumentação e pensamento crítico na enfermagem. 
Os dados revelam que os cursos de pós-graduação em enfermagem se preocupam em estudar aspectos históricos e as bases da filosofia, por meio de correntes de pensamento associadas ao processo de cuidar, à ética e ao raciocínio em enfermagem, permeando a teoria e a prática da profissão.

Nesse contexto, a enfermagem apropriou-se de alguns conceitos da área da filosofia para sustentar a fundamentação do cuidar, no intuito de explicar algumas inconsistências relacionadas às tarefas de cunho técnico e dependentes do modelo biomédico. Assim, pode-se realizar uma releitura na forma de fazer e pensar o cuidar. Neste sentido, espera-se que o estudo da filosofia possa contribuir para a fundamentação da razão no pensar, sentir, dizer e agir da enfermagem, por meio da análise da teoria e da práxis do cuidar (Waldow, 2015).

A formação do profissional da saúde é dinâmica, contínua e sofre influência do contexto social, político e econômico que a sociedade vivencia (Rodrigues et al., 2016). A Universidade tem papel ímpar nessas modificações e avanços, por isso é necessário desenvolver projetos pedagógicos que preparem profissionais, sobretudo com formação multiprofissional, para atender as demandas da sociedade, do ensino e da pesquisa nas universidades públicas e do Sistema Único de Saúde, considerando os diferentes perfis sociais e atuando de maneira crítica e reflexiva (Heck et al., 2016; Padovi; Corrêa, 2017).

$\mathrm{O}$ ensino da filosofia reflete na prática do filosofar. $\mathrm{Na}$ Universidade, pensar filosofia para aqueles que não sejam propriamente filósofos é um desafio; porém, é uma oportunidade para desenvolver o pensamento, que é livre e criativo. Todavia, é preciso levar em consideração o contexto acadêmico em que estamos inseridos, uma vez que determinados campos da educação não oferecem disciplinas de filosofia na grade curricular, e quando o fazem, por vezes é numa forma introdutória e dispersa (Rodrigues; Gonçalves; Ferreira, 2018). Essa realidade de subutilização da filosofia nas grades curriculares também foi evidenciada durante o processo de seleção deste estudo nas disciplinas nos programas de pós-graduação em enfermagem.

$\mathrm{O}$ estudo reflete que, em tempos de revisões curriculares e elaboração de novos projetos pedagógicos, a pergunta "por que a filosofia?" é importante, visto que não está pautada somente em entender como funciona seu ensino numa grade curricular de cursos, mas em lançar mão da filosofia para compreender o uso da razão, que há muito tempo vem sendo substituída pela racionalidade tecnicista. Ou seja, trata-se de refletir sobre a necessidade da filosofia em modelos de formação acadêmica (Rodrigues; Gonçalves; Ferreira, 2018).

A formação do enfermeiro e suas bases teórico-filosóficas vêm sendo debatidas desde o nível de graduação. Os debates são baseados na prática de enfermagem, metodologias de ensino e avaliações. Ainda, para qualificar a formação dos profissionais de enfermagem, as IES são instigadas a promover um ensino em que o discente consiga adaptar-se às mudanças sociais, tenha sensibilidade para construir propostas de relevância que tragam ganhos e transformações para população, baseadas em valores éticos e humanísticos, de forma igualitária e que respeite o seu papel social e político (Garanhani, 2016).

As limitações deste estudo residem na restrição das buscas por instituições públicas, excluindo as demais instituições, e ao contexto da enfermagem, restringindo-se, ainda, na modalidade acadêmica dos cursos de pós-graduação. Além disso, a indisponibilidade de informações referentes aos PE das disciplinas ofertadas pelas IES também dificultou o processo de coleta dos dados.

Entretanto, mesmo considerando os limites do estudo, os resultados obtidos mostram uma real e contemporânea fragilidade no que diz respeito aos aspectos de formação dos pós-graduando em enfermagem na área do saber da filosofia.

Além de despertarem uma reflexão sobre as bases filosóficas nas grades curriculares da pós-graduação em enfermagem, os resultados aqui obtidos também contribuem ao alicerçar possibilidades para futuras pesquisas. Sugere-se que outros estudos sejam conduzidos contemplando todos os programas de pós-graduação em enfermagem, na modalidade acadêmica e profissional, de todas as instituições no Brasil, não somente com intuito de mapear as grades curriculares, mas também buscando obter uma compreensão mais ampla da forma como têm sido conduzidas as abordagens filosóficas, e portanto, a sustentação crítica e reflexiva da formação do profissional enfermeiro pós-graduado. 


\section{Considerações Finais}

Com a realização deste estudo foi possível evidenciar a subutilização do campo de conhecimento da filosofia nos Planos de Ensino (PE) de programas de pós-graduação de enfermagem no Brasil, o que pode ser consequência da indisponibilidade de informações referentes aos PE das disciplinas ofertadas pelas IES.

Entretanto, cumpre salientar que os programas que abordam a temática apresentam uma preocupação em discutir com os discentes os aspectos conceituais, históricos e as correntes de pensamento, associadas ao processo de cuidar, à ética e ao raciocínio na enfermagem. Sendo assim, a abordagem de concepções filosóficas na pós-graduação em enfermagem pode contribuir para abrir novas fronteiras do conhecimento à profissão, além de estimular o raciocínio clínico no tocante aos aspectos teóricos e práticos da profissão.

Investir em disciplinas que abordam concepções filosóficas nos PPGENF é ter o compromisso de fortalecer um ensino baseado no saber científico, de forma crítica e reflexiva, formando profissionais com responsabilidade social e focado no processo de viver humano e nas ações de enfermagem.

Esperamos que o estudo sensibilize os organizadores dos programas de pós-graduação de enfermagem para adotar disciplinas com abordagem filosófica, pois o questionamento característico do agir filosófico confronta as práticas que já estão automatizadas. Entendemos que assim é possível instigar a construção de novos conhecimentos e avançar na consolidação da enfermagem como ciência e arte.

Diante do exposto sugere-se novos estudos acerca das abordagens filosóficas nos programas de pós-graduação em âmbito nacional, a fim de ampliar e fortalecer o pensamento crítico-reflexivo na formação de futuros pós-graduandos em enfermagem.

\section{Referências}

Barbosa, L. R., Reginato, V., \& Gallian, D. M. C. (2015). Literatura e o ensino da filosofia na graduação em enfermagem. Revista International de Salud, Bienestar y Soliedad, 2(1).

Cohen, J. (1960). A coefficient of agreement for nominal scale. Educational and Psychological Measurement.

Delors, J., Al-Mufti, I., Amagi, I., Carneiro, R., Chung, F., Geremek, B., et al. (1998). Educação um tesouro a descobrir. Relatório para a UNESCO da Comissão Internacional sobre Educação para o século XXI. Brasília: UNESCO.

Garanhani, M. L. (2016). Os desafios na formação do enfermeiro no século XXI. R. Enferm. Cent. O. Min., 6(3). http://www.seer.ufsj.edu.br/index.php/recom/article/view/1638/1163

Guimarães, G. L., Chianca, T. C. M., Goveia, V. R., Mendoza, I. Y. Q., Matos, S. S., \& Viana L. O. (2017). A contribuição de Imre Lakatos para a análise epistemológica do programa brasileiro de pós-graduação em enfermagem. Texto \& contexto enferm, 26(1):e384001. http://www.redalyc.org/pdf/714/71449839023.pdf

Heck, R. M., Thumé, E., Schwartz, E., Buss, E., Lise, F., \& Kessler, M. (2016). Fragmentos da história da pós-graduação em enfermagem da Universidade Federal de Pelotas. J Nurs Health, 6(suppl.):211-23.

Landis, J. R., \& Koch, G. G. (1977). The measurement of observer agreement for categorical data. Biometrics; 33(1):159-174. https://www.jstor.org/stable/2529310?seq=1\#metadata_info_tab_contents

Liberatori, R. S. M. (2019). Professores e o ensino do pensamento crítico em enfermagem: por uma abordagem da filosofia da linguagem. Rev Fermentario, 13(1). https://ojs.fhce.edu.uy/index.php/fermen/article/view/523/462

Mcewen, M., Wills, E. M. (2015). Bases Teóricas de Enfermagem. (4a ed.), Artmed Editora.

Merino, M. F. G. L, Silva, P. L. A. R., Carvalho, M. D. B., Pelloso, S. M., Baldissera, V. D. A., \& Higarashi, I. H. (2018). Teorias de enfermagem na formação e na prática profissional: percepção dos pós-graduandos de enfermagem. Rev. Rene, 19(1):1-8. http://www.periodico s.ufc.br/rene/article/view/32803/pdf_1

Padovani, O., \& Corrêa, A. K. (2017). Currículo e formação do enfermeiro: desafios das universidades na atualidade. Sau. \& Transf. Soc., 8(2):112-19.

Rodrigues, C. C. F. M., Carvalho, D. P. S. R. P., Salvador, P. T. C. O., Medeiros, S. M., Menezes, R. M. P., Ferreira Júnior, M. A., \& Pereira, V. E. (2016). Innovative nursing education from the perspective of epistemologies of the South. Escola Anna Nery, 20(2), 384-389. https://doi.org/10.5935/14148145.20160053 
Research, Society and Development, v. 10, n. 4, e18610414073, 2021

(CC BY 4.0) | ISSN 2525-3409 | DOI: http://dx.doi.org/10.33448/rsd-v10i4.14073

Rodrigues, V. F., Gonçalves, M. D., \& Ferreira, A. R. S. (2018). O lugar e importância da filosofia na formação docente. Problemata: R. Intern. Fil., 9(3):28693.

Salcedo-Álvarez, R. A. (2017). El profesional de enfermería que México necesita. Rev Enferm Inst Mex Seguro Soc., 25(1):75-9. https://www.medigraphic.com/pdfs/enfermeriaimss/eim-2017/eim171m.pdf

Salviano, M. E. M., Nascimento, P. D. F. S., Paula, M. A., Vieira, C. S., Frison, S. S., Maia, M. A., Souza, K. V., \& Borges, E. L. (2016). Epistemologia do cuidado de enfermagem: uma reflexão sobre suas bases. Revista Brasileira de Enfermagem, 69(6), 1240-1245. https://doi.org/10.1590/0034-7167-2016-0331

Schmitz, E. L., Gelbcke, F. L., Bruggmann, M. S., \& Luz, S. C. L. (2016). Filosofia e marco conceitual: estruturando coletivamente a sistematização da assistência de enfermagem. Revista Gaúcha de Enfermagem, 37, e68435. https://doi.org/10.1590/1983-1447.2016.esp.68435

Sousa, F. G. M., Koerich, M. S. (Org.) (2008). Cuidar-cuidado: reflexões contemporâneas. Papa-Livro.

Waldow, V. R. (2015). Enfermagem: a prática do cuidado sob o ponto de vista filosófico. Investigación en Enfermería: Imagen y Desarrollo, 17(1):13-25. https://www.redalyc.org/articulo.oa?id=145233516002 